\title{
PROPAGATION OF UNIFORM GEVREY REGULARITY OF SOLUTIONS TO EVOLUTION EQUATIONS
}

\author{
TODOR GRAMCHEV \\ Dipartimento di Matematica \\ Università di Cagliari \\ 09124 Cagliari, Italy \\ E-mail:todor@unica.it \\ YA-GUANG WANG \\ Department of Mathematics \\ Shanghai Jiao Tong University \\ 200030 Shanghai, P.R. China \\ E-mail: ygwang@sjtu.edu.cn
}

\begin{abstract}
We investigate the propagation of the uniform spatial Gevrey $G^{\sigma}, \sigma \geq 1$, regularity for $t \rightarrow+\infty$ of solutions to evolution equations like generalizations of the Euler equation and the semilinear Schrödinger equation with polynomial nonlinearities. The proofs are based on direct iterative arguments and nonlinear Gevrey estimates.
\end{abstract}

1. Introduction. The main goal of this paper is to investigate the propagation for $t \rightarrow+\infty$ of the uniform Gevrey regularity with respect to the space variables $x$ of solutions to Cauchy problems for some classes of nonlinear evolution equations by means of techniques, based on iterative arguments and nonlinear estimates in the framework of Gevrey spaces. The initial data are supposed to be uniformly $G^{\sigma}(\Omega)$ Gevrey (we write shortly $\left.v \in G_{u n}^{\sigma}(\Omega)\right)$ for some $\sigma \geq 1$, where $\Omega=\mathbb{R}^{n}$ or $\Omega$ is the $n$-dimensional torus $\mathbb{T}^{n}=\mathbb{R}^{n} /(2 \pi \mathbb{Z})^{n}$. More precisely, $v \in G_{u n}^{\sigma}(\Omega)$ means that there exists $\rho>0$ such that

$$
\|v\|_{\sigma, \rho, H^{r}}:=\sup _{\alpha \in \mathbb{Z}_{+}^{n}}\left(\frac{\rho^{|\alpha|}\left\|\partial^{\alpha} v\right\|_{H^{r}}}{(\alpha !)^{\sigma}}\right)<+\infty,
$$

Research of the authors is partially supported by GNAMPA-INDAM, Italy. The research of the first author is supported in part by local research projects of the University of Cagliari. The research of the second author is supported in part by the NSFC and the Education Ministry of China.

2000 Mathematics Subject Classification: Primary 35B65; Secondary 35G25, 35S05.

The paper is in final form and no version of it will be published elsewhere. 
where $\alpha !=\alpha_{1} ! \cdots \alpha_{n} !, \alpha=\left(\alpha_{1}, \ldots, \alpha_{n}\right) \in \mathbb{Z}_{+}^{n}, r \geq 0$ is an integer, and $H^{r}=H^{r}(\Omega)=$ $W^{r, 2}(\Omega)$ stands for the classical $L^{2}$ based Sobolev space of order $r$. Define $G^{\sigma}\left(\Omega, H^{r}, \rho\right)$, $\rho>0$, as the space of all functions $v \in C^{\infty}(\Omega)$ satisfying (1.1). We note that $G^{1}\left(\Omega, H^{r}, \rho\right)$ is a subset of the space of uniformly analytic functions in $\Omega$ and every $v \in G^{1}\left(\Omega, H^{r}, \rho\right)$ can be extended to a holomorphic function in the strip $\left\{z \in \mathbb{C}^{n}:|\operatorname{Im} z|<\rho\right\}$. As in Proposition 1.4.5 of [Ro], we have that $G^{\sigma}\left(\Omega, H^{r}, \rho\right)$ is a vector space and, if $r>n / 2$, a ring with respect to the arithmetic product of functions, and is closed under differentiation. We refer to the book [Ro] for the basic theory of Gevrey spaces.

In view of the lack of space we will focus on two particular classes of equations. The first one is a generalization of the incompressible Euler equation (cf. [LO]). We will extend the results in [LO] for the propagation of the analytic regularity on $\mathbb{T}^{2}$ in the framework of all Gevrey classes $G^{\sigma}, \sigma \geq 1$. More precisely, we consider the Cauchy problem

$$
\begin{aligned}
\partial_{t} \omega+K[\omega] \cdot \nabla \omega & =0, & & t>0, x \in \Omega \\
\omega(0, x) & =\omega^{0}(x), & & x \in \Omega
\end{aligned}
$$

where $\Omega=\mathbb{R}^{n}$ or $\Omega=\mathbb{T}^{n}$, and the operator $K$ satisfies the following hypotheses:

(H1) $K$ is linear: $H^{s}\left(\mathbb{R}^{n}\right) \ni \omega \longrightarrow K[\omega]=\left(K_{1}[\omega], \ldots, K_{n}[\omega]\right) \in\left(H^{s+1}(\Omega)\right)^{n}, s \geq 0$;

(H2) there exists a real valued function $b \in G_{u n}^{\sigma}(\Omega), \inf _{x \in \Omega} b(x)=: b_{0}>0$ such that

$$
\nabla \cdot(b K[\omega])=0, \quad \omega \in H^{s}(\Omega), s \geq 0 ;
$$

(H3) for any $s \geq 0, K$ is bounded from $H^{s}(\Omega)$ to $\left(H^{s+1}(\Omega)\right)^{n}$, namely there exists a positive constant $C$ such that

$$
\|K[\omega]\|_{H^{s+1}} \leq C\|\omega\|_{H^{s}}, \quad \omega \in H^{s}(\Omega) .
$$

The hypotheses (H1)-(H3) are satisfied for generalizations of the Euler equation on the two-dimensional torus related to shallow water equations, cf. [LO]. For the Euler equation in a bounded domain in $\mathbb{R}^{2}$ with smooth real analytic boundary, the propagation of the uniform analyticity of solutions to the Dirichlet boundary value problem has been investigated in $[\mathrm{BB}]$ by means of subtle estimates on the Green function of the Poisson kernel in complex analytic neighbourhoods of the domain. The propagation of the local analyticity of solutions to the Euler equation in $\mathbb{R}^{n}$ was studied in $[\mathrm{AM}]$. Recently, the propagation of the uniform analyticity of solutions to Euler type equations on the torus $\mathbb{T}^{n}$ was investigated in [LO] by means of nonlinear estimates in Gevrey $G^{1}$ spaces and the Galerkin approximation (cf. also [BG], [FT], [FeT] for results on analytic regularity for dissipative equations like the Navier-Stokes equation for incompressible fluids, the Kuramoto-Sivashinsky equation, and semilinear parabolic equations). Theorems on the existence and/or uniqueness of classical solutions to the initial value problem for Euler type equations can be found in $[\mathrm{Ba}],[\mathrm{Ka}],[\mathrm{LOT}],[\mathrm{Ol}]$.

Another motivation for our investigations comes from results on the propagation of the local analytic and, more generally, local $G^{\sigma}$ Gevrey regularity for linear and nonlinear hyperbolic equations (e.g., cf. [CZ], [Kj1], [Kj2], [KY2], [RY], [Sp] and references therein). We mention also that uniformly analytic functions in $\mathbb{R}^{n}$ as initial data have been used as a functional set-up for proving global well-posedness of initial value problems for degenerate Kirchhoff type equations (e.g., cf. [DS], [KY1]). 
The second type of equations will be a model semilinear Schrödinger equation

$$
\begin{aligned}
i \partial_{t} u+\Delta u+g(u) & =0, & & t>0, x \in \mathbb{R}^{n} \\
u(0, x) & =u_{0}(x), & & x \in \mathbb{R}^{n},
\end{aligned}
$$

where $g$ is a polynomial, vanishing of order $q \geq 2$ at the origin, i.e.

$$
g(u)=\sum_{\beta=q}^{p} g_{\beta} u^{\beta}, \quad g_{\beta} \in \mathbb{C}, \beta=q, \ldots, p, g_{q} \neq 0
$$

for some integer $p \geq q$.

As a corollary from more general results (cf. $[\mathrm{KP}]$, see also $[\mathrm{Ra}]$ ) we know that under the assumption

$$
\frac{q}{(q-1)^{2}}<\frac{n}{2}
$$

there exists an integer $r_{0}>n / 2+2$, depending only on the dimension $n$, and a small constant $\delta_{0}>0$ such that if

$$
u_{0} \in H^{r}\left(\mathbb{R}^{n}\right) \cap W^{r, p}\left(\mathbb{R}^{n}\right), \quad p=\frac{2 q}{2 q-1}
$$

for $r \in \mathbb{N}, r \geq r_{0}$, and

$$
\left\|u_{0}\right\|_{H^{r}}+\left\|u_{0}\right\|_{W^{r, p}} \leq \delta, \quad 0 \leq \delta \leq \delta_{0},
$$

then the Cauchy problem (1.6), (1.7) admits a unique classical solution

$$
u \in C\left(\left[0,+\infty\left[: H^{r}\left(\mathbb{R}^{n}\right)\right) \cap C^{1}\left(\left[0,+\infty\left[: H^{r-2}\left(\mathbb{R}^{n}\right)\right) .\right.\right.\right.\right.
$$

Moreover, for $t \rightarrow+\infty$, we have

$$
\|u(t, \cdot)\|_{L^{\kappa}}=O\left(t^{-\gamma}\right), \quad \gamma=\frac{n(q-1)}{2 q}, \quad 2 q \leq \kappa \leq+\infty,
$$

and there exists a constant $M_{0}$ depending on $\delta_{0}$ and $r$ such that

$$
\|u(t, \cdot)\|_{H^{r}} \leq M_{0}\left\|u_{0}\right\|_{H^{r}}, \quad t \geq 0 .
$$

Similar results for global in time solutions for small data are available for nonlinear wave equations and the Klein-Gordon equation (cf. $[\mathrm{KP}]$, $[\mathrm{Ra}]$, see also $[\mathrm{GG}]$ for extensions to certain quasilinear weakly hyperbolic systems).

We state the first main result of our paper for the initial value problem (1.2)-(1.3) under the assumptions (H1)-(H3).

THEOREM 1. Let us fix $r \in \mathbb{N}, r>n / 2+1$ and $\sigma \geq 1$. Then we can find a positive constant $c_{0}$, depending only on $n, r$ and the function $b$, such that for every $\omega^{0} \in G^{\sigma}\left(\Omega, H^{r}, \rho\right)$, $\rho>0$, the unique global solution $\omega \in C\left(\left[0,+\infty\left[: H^{r}(\Omega)\right)\right.\right.$ to $(1.2),(1.3)$ satisfies

$$
\omega(t, \cdot) \in G^{\sigma}\left(\Omega, H^{r}, \rho_{\sigma}(t)\right),
$$

where

$$
\rho_{\sigma}(t)=\rho \exp \left(-c_{0}\left(t\left\|\omega^{0}\right\|_{\sigma, \rho, H^{r}}+\int_{0}^{t}\left\|\nabla K\left[\omega\left(t_{1}, \cdot\right)\right]\right\|_{H^{r}} d t_{1}\right)\right),
$$

for all $t \geq 0$. 
REMARK 1. We point out that if $\Omega=\mathbb{T}^{2}$ and $\sigma=1$, the decay of the Gevrey $G^{1}$ radius $\rho_{1}(t)$ in $(1.16)$ is slower than the decay rate in Theorem 7 of [LO]. We refer to Section 5 for more details.

Next, we investigate the propagation of the uniform Gevrey regularity of solutions to the Schrödinger equation (1.6).

Theorem 2. Assume that (1.9) holds and choose and fix $r \in \mathbb{N}, r \geq r_{0}>n / 2+2$ and a Gevrey index $\sigma \geq 1$. Let $\delta_{0}>0$ be as in (1.11) and let $u_{0}$ satisfy (1.10), (1.11) and $u_{0} \in G^{\sigma}\left(\mathbb{R}^{n}, H^{r}, \rho\right)$ for some $\rho>0$. Then the unique classical solution $u$ of $(1.6),(1.7)$ satisfies

$$
u(t, \cdot) \in G^{\sigma}\left(\mathbb{R}^{n}, H^{r}, \rho_{\sigma}(t)\right), \quad t \geq 0
$$

where

$$
\rho_{\sigma}(t)=\rho \exp \left(-\lambda_{0}\left(\left\|u_{0}\right\|_{\sigma, \rho, H^{r}}\right)^{q-1} t-c_{0}(1+t)^{1-\gamma(q-1)}\right), \quad t \geq 0,
$$

while $c_{0}>0$ and $\lambda_{0}$ are constants depending only on $\delta_{0}, r, n, M_{0}$ and the coefficients of the polynomial $\mathrm{g}$. Moreover, for every $0<\varepsilon \ll 1$ we can find $0<\delta(\varepsilon) \ll 1$ and $\widetilde{c}_{0}>0$ such that

$$
\rho_{\sigma}(t)=\rho \exp \left(-\varepsilon t-\widetilde{c_{0}}(1+t)^{1-\gamma(q-1)}\right), \quad t \geq 0
$$

provided $u_{0}$ satisfies (1.11) with $\delta=\delta(\varepsilon)$.

The paper is organized as follows. In Section 2 we give the proof of Theorem 1 provided certain nonlinear Gevrey estimates are satisfied. We derive the nonlinear estimates in Section 3. The proof of Theorem 2 is carried out in Section 4. Finally, in Section 5, we discuss relations of our theorems with previous results and outline possible generalizations.

\section{Proof of Theorem 1. Let}

$$
\begin{aligned}
Z_{k}(t) & =\|\omega(t, \cdot)\|_{H^{r+k}}, \\
u(t, x) & =K[\omega(t, \cdot](x) .
\end{aligned}
$$

In the sequel, we will use $C_{*}$ to denote any absolute constant independent of $k \in \mathbb{N}$ and $\omega, *$ being an index.

The crucial ingredient in the proof of Theorem 1 is the following estimate, which will be proven in the next section:

\section{PROPOSITION 3 .}

1. If $k \geq r+2$, then

$$
\begin{aligned}
\frac{d}{d t} Z_{k}(t) \leq C_{0}\left(k\|u(t, \cdot)\|_{H^{r}} Z_{k}(t)\right. & +\sum_{j=2}^{N}\left(\begin{array}{c}
r+k \\
j
\end{array}\right) Z_{j-1}(t) Z_{k+1-j}(t) \\
& \left.+\sum_{j=N+1}^{r+k}\left(\begin{array}{c}
r+k \\
j
\end{array}\right) Z_{j-r-1}(t) Z_{k+r+1-j}(t)\right)
\end{aligned}
$$

where $N=\left[\frac{r+k}{2}\right]$ is the integer part of $\frac{r+k}{2}$. 
2. If $k \leq r+1$, then

$$
\begin{aligned}
& \frac{d}{d t} Z_{k}(t) \leq C_{0}\left(k\|u(t, \cdot)\|_{H^{r}} Z_{k}(t)+\sum_{j=2}^{k}\left(\begin{array}{c}
r+k \\
j
\end{array}\right) Z_{j-1}(t) Z_{k+1-j}(t)\right. \\
& \left.\quad+\sum_{j=k+1}^{r+1}\left(\begin{array}{c}
r+k \\
j
\end{array}\right) Z_{1}(t) Z_{k-1}(t)+\sum_{j=r+2}^{r+k}\left(\begin{array}{c}
r+k \\
j
\end{array}\right) Z_{j-r-1}(t) Z_{k+r+1-j}(t)\right),
\end{aligned}
$$

where the third term does not appear if $k=r+1$.

Now, we want to conclude the assertion of Theorem 1 from the estimates (2.3) and (2.4).

We set

$$
P(t)=C_{0} \int_{0}^{t}\|u(s, \cdot)\|_{H^{r}} d s
$$

and

$$
\Theta_{k}(t)=\frac{\epsilon_{k}}{(k !)^{\sigma}} e^{-k P(t)} Z_{k}(t)
$$

where $\epsilon_{k}$ will be determined later.

When $k \geq r+2$, from (2.3) we have

$$
\begin{aligned}
& \Theta_{k}^{\prime}(t)=\frac{\epsilon_{k}}{(k !)^{\sigma}} e^{-k P(t)}\left(Z_{k}^{\prime}(t)-C_{0} k\|u(t, \cdot)\|_{H^{r}} Z_{k}(t)\right) \\
& \leq \frac{C_{0} \epsilon_{k}}{(k !)^{\sigma}} e^{-k P(t)}\left(\sum_{j=2}^{N}\left(\begin{array}{c}
r+k \\
j
\end{array}\right) Z_{j-1}(t) Z_{k+1-j}(t)\right. \\
&\left.\quad+\sum_{j=N+1}^{r+k}\left(\begin{array}{c}
r+k \\
j
\end{array}\right) Z_{j-r-1}(t) Z_{k+r+1-j}(t)\right) .
\end{aligned}
$$

Obviously, from (2.6) we have

$$
Z_{j-1}(t) Z_{k+1-j}(t)=\left(\begin{array}{c}
k \\
j-1
\end{array}\right)^{-\sigma} \frac{(k !)^{\sigma}}{\epsilon_{j-1} \epsilon_{k+1-j}} e^{k P(t)} \Theta_{j-1}(t) \Theta_{k+1-j}(t) .
$$

Substituting (2.8) into (2.7) we get

$$
\begin{aligned}
& \Theta_{k}^{\prime}(t) \leq C_{0}\left(\sum_{j=2}^{N}\left(\begin{array}{c}
r+k \\
j
\end{array}\right)\left(\begin{array}{c}
k \\
j-1
\end{array}\right)^{-\sigma} \frac{\epsilon_{k}}{\epsilon_{j-1} \epsilon_{k+1-j}} \Theta_{j-1}(t) \Theta_{k+1-j}(t)\right. \\
& \left.+\sum_{j=N+1}^{r+k}\left(\begin{array}{c}
r+k \\
j
\end{array}\right)\left(\begin{array}{c}
k \\
j-r-1
\end{array}\right)^{-\sigma} \frac{\epsilon_{k}}{\epsilon_{j-r-1} \epsilon_{k+r+1-j}} \Theta_{j-r-1}(t) \Theta_{k+r+1-j}(t)\right) .
\end{aligned}
$$

By using in (2.9) the relations

$$
\left(\begin{array}{c}
r+k \\
j
\end{array}\right)\left(\begin{array}{c}
k \\
j-1
\end{array}\right)^{-\sigma} \leq C_{1} k\left(\begin{array}{c}
k \\
j-1
\end{array}\right)^{-\sigma+1} \leq C_{1} k
$$

when $2 \leq j \leq\left[\frac{r+k}{2}\right]$, and

$$
\left(\begin{array}{c}
r+k \\
j
\end{array}\right)\left(\begin{array}{c}
k \\
j-r-1
\end{array}\right)^{-\sigma} \leq C_{2}\left(\begin{array}{c}
k \\
j-r-1
\end{array}\right)^{-\sigma+1} \leq C_{2}
$$


when $j \geq\left[\frac{r+k}{2}\right]+1$, we obtain

$$
\begin{aligned}
\Theta_{k}^{\prime}(t) \leq C_{3}\left(k \sum_{j=2}^{N} \frac{\epsilon_{k}}{\epsilon_{j-1} \epsilon_{k+1-j}} \Theta_{j-1}(t) \Theta_{k+1-j}(t)\right. \\
\left.\quad+\sum_{j=N+1}^{r+k} \frac{\epsilon_{k}}{\epsilon_{j-r-1} \epsilon_{k+r+1-j}} \Theta_{j-r-1}(t) \Theta_{k+r+1-j}(t)\right) .
\end{aligned}
$$

If we choose $\epsilon_{k}=k^{2}$, then we have

$$
\begin{gathered}
\sum_{j=2}^{N} \frac{\epsilon_{k}}{\epsilon_{j-1} \epsilon_{k+1-j}}=\sum_{j=2}^{N}\left(\frac{1}{j-1}+\frac{1}{k+1-j}\right)^{2} \leq C_{4}, \\
\sum_{j=N+1}^{r+k} \frac{\epsilon_{k}}{\epsilon_{j-r-1} \epsilon_{k+r+1-j}}=\sum_{j=N+1}^{r+k}\left(\frac{1}{j-r-1}+\frac{1}{k+r+1-j}\right)^{2} \leq C_{5},
\end{gathered}
$$

with $C_{4}, C_{5}$ being independent of $k \in \mathbb{N}$.

Thus, from (2.10) we obtain

$$
\Theta_{k}^{\prime}(t) \leq C_{6} k \max _{1 \leq j \leq k-1} \Theta_{j}(t) \Theta_{k-j}(t),
$$

where $C_{6}$ is an absolute constant depending on $r>1$.

When $k \leq r+1$, it is not difficult to derive from (2.4) that we also have the estimate (2.11) by noting that all coefficients on the right-hand side of (2.4) are bounded by a constant depending only on $r$.

If we set

$$
\mu_{k}^{\lambda}(t):=e^{-\lambda k t} \Theta_{k}(t)=\frac{k^{2}}{(k !)^{\sigma}} e^{-k(\lambda t+P(t))}\|\omega(t, \cdot)\|_{H^{r+k}}
$$

with $P(t)$ being given in (2.5), then, from (2.11) and from the choice of the initial datum $\omega^{0} \in G^{\sigma}\left(\Omega, H^{r}, \rho\right)$ we obtain

$$
\begin{aligned}
\mu_{k}^{\lambda}(t) \leq \mu_{k}^{\lambda}(0)+\frac{C_{6}}{\lambda} \max _{1 \leq j \leq k-1} \max _{0 \leq s \leq t} \mu_{j}^{\lambda}(s) \mu_{k-j}^{\lambda}(s) \\
\quad \leq\left\|\omega^{0}\right\|_{\sigma, \rho, H^{r}}+\frac{C_{6}}{\lambda} \max _{1 \leq j \leq k-1} \max _{0 \leq s \leq t} \mu_{j}^{\lambda}(s) \mu_{k-j}^{\lambda}(s) .
\end{aligned}
$$

Set

$$
\Gamma_{N}(\lambda):=\max _{1 \leq k \leq N} \sup _{t \geq 0} \mu_{k}^{\lambda}(t), \quad N=1,2, \ldots
$$

Since $\Gamma_{0}(\lambda)<+\infty$, we are reduced to the following sequence of iteration inequalities

$$
\Gamma_{N}(\lambda) \leq\left\|\omega^{0}\right\|_{\sigma, \rho, H^{r}}+\frac{C_{6}}{\lambda}\left(\Gamma_{N-1}(\lambda)\right)^{2}, \quad N=1,2, \ldots
$$

The sequence $\left\{\Gamma_{N}^{\lambda}\right\}_{N=1}^{\infty}$ converges provided $\lambda \geq \lambda_{0}:=4 C_{6}\left\|\omega^{0}\right\|_{\sigma, \rho, H^{r}}$. Choosing $\lambda=\lambda_{0}$ we get the estimate

$$
\Gamma_{N}^{\lambda_{0}} \leq 2\left\|\omega^{0}\right\|_{\sigma, \rho, H^{r}}, \quad N=1,2, \ldots,
$$

which leads to

$$
\mu_{k}^{\lambda_{0}}(t) \leq 2\left\|\omega^{0}\right\|_{\sigma, \rho, H^{r}}
$$

for all $t \geq 0, k \in \mathbb{N}$. The proof of Theorem 1 is complete. 
3. Nonlinear Gevrey estimates. We will use the notation $\hat{f}(\xi)=\mathcal{F}_{x \rightarrow \xi} f$ for the discrete Fourier transformation

$$
\hat{f}(\xi):=\int_{\mathbb{T}^{n}} e^{-i x \xi} f(x) d x, \quad \xi \in \mathbb{Z}^{n},
$$

in the case $\Omega=\mathbb{T}^{n}$ while if $\Omega=\mathbb{R}^{n}$ we will rely on the continuous Fourier transformation

$$
\hat{f}(\xi)=\int_{\mathbb{R}^{n}} e^{-i x \xi} f(x) d x, \quad \xi \in \mathbb{R}^{n} .
$$

First of all, we have

Lemma 4. Let $r \in \mathbb{N}, r>n / 2$ be fixed, $\omega$ be the classical solution to the IVP (1.2), (1.3), and let $A$ be the positive and self-adjoint operator $A:=\left(1-\partial_{x_{1}}^{2}-\ldots-\partial_{x_{n}}^{2}\right)^{1 / 2}$. Then for any $k \in \mathbb{N}, \omega \in C\left(\left[0,+\infty\left[: H^{+\infty}(\Omega)\right)\right.\right.$ we have

$$
\left|\left\langle u \cdot \nabla\left(A^{r+k} \omega\right), A^{r+k} \omega\right\rangle(t)\right| \leq C_{0}\|u(t, \cdot)\|_{L^{\infty}}\|\omega(t, \cdot)\|_{H^{r+k}(\Omega)}^{2}
$$

where $u=K[\omega]($ as in $(2.2)), H^{+\infty}(\Omega)=\bigcap_{s \geq 0} H^{s}(\Omega)$,

$$
\langle\mu, \nu\rangle(t):=\int_{\Omega} \mu(t, x) \overline{\nu(t, x)} d x, \quad \mu, \nu \in C\left(\left[0,+\infty\left[: L^{2}(\Omega)\right),\right.\right.
$$

and $C_{0}=2^{-1} b_{0}^{-1}\|\nabla b\|_{L^{\infty}}, b_{0}>0$ being defined in the assumption (H2).

Proof. By using $\nabla \cdot(b u)=0, \nabla b^{-1}=-b^{-2} \nabla b$, and the definition of $b_{0}>0$ in (H2), we have

$$
\begin{aligned}
\left|\left\langle u \cdot \nabla\left(A^{r+k} \omega\right), A^{r+k} \omega\right\rangle(t)\right|=\left|\frac{1}{2}\left\langle\left(A^{r+k} \omega\right)^{2}, \nabla \cdot u\right\rangle(t)\right| & \\
=\mid \frac{1}{2}\left\langle\left(A^{r+k} \omega\right)^{2}\right. & \left.,\left(\nabla b^{-1}\right) \cdot b u\right\rangle(t) \mid \\
& \leq \frac{\|\nabla b\|_{L^{\infty}}}{2 b_{0}}\|u(t, \cdot)\|_{L^{\infty}}\|\omega(t, \cdot)\|_{H^{r+k}(\Omega)}^{2}
\end{aligned}
$$

which implies the desired inequality (3.3).

The main ingredient in the proof of Proposition 3 is to establish

Proposition 5.

1. With the same notation as above, if $k \geq r+2$, then

$$
\begin{aligned}
\mid\left\langle u \cdot \nabla\left(A^{r+k} \omega\right), A^{r+k} \omega\right\rangle(t)- & \left\langle A^{r+k}(u \cdot \nabla \omega), A^{r+k} \omega\right\rangle(t) \mid \\
\leq C_{0} Z_{k}(t)\left(k\|u(t, \cdot)\|_{H^{r}} Z_{k}(t)\right. & +\sum_{j=2}^{N}\left(\begin{array}{c}
r+k \\
j
\end{array}\right) Z_{j-1}(t) Z_{k+1-j}(t) \\
& \left.+\sum_{j=N+1}^{r+k}\left(\begin{array}{c}
r+k \\
j
\end{array}\right) Z_{j-r-1}(t) Z_{k+r+1-j}(t)\right)
\end{aligned}
$$

where $Z_{k}(t)=\|\omega(t, \cdot)\|_{H^{r+k}}$ and $N=\left[\frac{r+k}{2}\right]$. 
2. If $k \leq r+1$, then

$$
\begin{aligned}
& \left|\left\langle u \cdot \nabla\left(A^{r+k} \omega\right), A^{r+k} \omega\right\rangle(t)-\left\langle A^{r+k}(u \cdot \nabla \omega), A^{r+k} \omega\right\rangle(t)\right| \\
& \leq C_{0} Z_{k}(t)\left(k\|u(t, \cdot)\|_{H^{r}} Z_{k}(t)+\sum_{j=2}^{k}\left(\begin{array}{c}
r+k \\
j
\end{array}\right) Z_{j-1}(t) Z_{k+1-j}(t)\right. \\
& \left.\quad+\sum_{j=k+1}^{r+1}\left(\begin{array}{c}
r+k \\
j
\end{array}\right) Z_{1}(t) Z_{k-1}(t)+\sum_{j=r+2}^{r+k}\left(\begin{array}{c}
r+k \\
j
\end{array}\right) Z_{j-r-1}(t) Z_{k+r+1-j}(t)\right),
\end{aligned}
$$

where the third term does not appear when $k=r+1$.

Proof. Set $\langle\xi\rangle=\left(1+|\xi|^{2}\right)^{1 / 2}, \xi$ being the dual variable of $x \in \Omega$. Clearly, we have

$$
\begin{aligned}
& \left|\left\langle u \cdot \nabla\left(A^{r+k} \omega\right), A^{r+k} \omega\right\rangle(t)-\left\langle A^{r+k}(u \cdot \nabla \omega), A^{r+k} \omega\right\rangle(t)\right| \\
& \left.\left.=\mid \int\langle\xi\rangle^{r+k} \overline{\hat{\omega}}(t, \xi) \widehat{\left(u \cdot \nabla \left(A^{r+k} \omega\right.\right.}\right)(t, \xi)-\langle\xi\rangle^{r+k} \widehat{u \cdot \nabla \omega}(t, \xi)\right) d \xi \mid \\
& \leq \iint\langle\xi\rangle^{r+k}|\hat{\omega}(t, \xi)| \sum_{j=1}^{r+k}\left(\begin{array}{c}
r+k \\
j
\end{array}\right)\langle\xi-\eta\rangle^{j} \\
& \times\langle\eta\rangle^{r+k+1-j}|\hat{u}(t, \xi-\eta)||\hat{\omega}(t, \eta)| d \eta d \xi
\end{aligned}
$$

by using

$$
\langle\xi\rangle^{r+k} \leq \sum_{j=0}^{r+k}\left(\begin{array}{c}
r+k \\
j
\end{array}\right)\langle\xi-\eta\rangle^{j}\langle\eta\rangle^{r+k-j} .
$$

For the term with $j=1$ in (3.7), we have due to assumption (H3)

$$
\begin{gathered}
\iint\langle\xi\rangle^{r+k}|\hat{\omega}(t, \xi)|\langle\xi-\eta\rangle\langle\eta\rangle^{r+k}|\hat{u}(t, \xi-\eta)||\hat{\omega}(t, \eta)| d \eta d \xi \\
=\iint \frac{\langle\xi\rangle^{r+k}|\hat{\omega}(t, \xi)|}{\langle\xi-\eta\rangle^{r}}\langle\xi-\eta\rangle^{r+1}\langle\eta\rangle^{r+k}|\hat{u}(t, \xi-\eta)||\hat{\omega}(t, \eta)| d \eta d \xi \\
\leq C_{0}\|u(t, \cdot)\|_{H^{r+1}} Z_{k}^{2}(t) \leq C_{1}\|\omega(t, \cdot)\|_{H^{r}} Z_{k}^{2}(t) .
\end{gathered}
$$

Denote by $I$ the sum of terms from $j=2$ to $r+k$ on the right-hand side of (3.7).

1. When $k \geq r+2$, we decompose $I$ into two parts as follows:

$$
\begin{aligned}
I=\sum_{j=2}^{r+k}\left(\begin{array}{c}
r+k \\
j
\end{array}\right) & \iint\langle\xi\rangle^{r+k}|\hat{\omega}(t, \xi)|\langle\xi-\eta\rangle^{j} \\
\times\langle\eta\rangle^{r+k+1-j}|\hat{u}(t, \xi-\eta)| & |\hat{\omega}(t, \eta)| d \eta d \xi \\
& =\left(\sum_{j=2}^{N}+\sum_{j=N+1}^{r+k}\right) \ldots=: I_{1}+I_{2},
\end{aligned}
$$

where $N=\left[\frac{r+k}{2}\right]$. 
For the term $I_{1}$ we have

$$
\begin{aligned}
& I_{1}=\sum_{j=2}^{N}\left(\begin{array}{c}
r+k \\
j
\end{array}\right) \iint\langle\xi\rangle^{r+k}|\hat{\omega}(t, \xi)|\langle\xi-\eta\rangle^{r+j}|\hat{u}(t, \xi-\eta)| \\
& \times \frac{\langle\eta\rangle^{r+k+1-j}|\hat{\omega}(t, \eta)|}{\langle\xi-\eta\rangle^{r}} d \eta d \xi \\
& \leq C_{2} \sum_{j=2}^{N}\left(\begin{array}{c}
r+k \\
j
\end{array}\right) Z_{k}(t) Z_{j-1}(t) Z_{k+1-j}(t) .
\end{aligned}
$$

For the term $I_{2}$ we have

$$
\begin{aligned}
I_{2}=\sum_{j=N+1}^{r+k}\left(\begin{array}{c}
r+k \\
j
\end{array}\right) & \iint \frac{\langle\xi\rangle^{r+k}|\hat{\omega}(t, \xi)|}{\langle\eta\rangle^{r}}\langle\xi-\eta\rangle^{j}|\hat{u}(t, \xi-\eta)| \\
& \times\langle\eta\rangle^{2 r+k+1-j}|\hat{\omega}(t, \eta)| d \eta d \xi \\
& \leq C_{3} \sum_{j=N+1}^{r+k}\left(\begin{array}{c}
r+k \\
j
\end{array}\right) Z_{k}(t) Z_{j-r-1}(t) Z_{k+r+1-j}(t) .
\end{aligned}
$$

Substituting (3.9)-(3.12) into (3.7), we conclude (3.5).

2. When $k \leq r+1$, we decompose $I$ into three parts as follows:

$$
\begin{gathered}
I=\sum_{j=2}^{r+k}\left(\begin{array}{c}
r+k \\
j
\end{array}\right) \iint\langle\xi\rangle^{r+k}|\hat{\omega}(t, \xi)|\langle\xi-\eta\rangle^{j} \\
\times\langle\eta\rangle^{r+k+1-j}|\hat{u}(t, \xi-\eta)||\hat{\omega}(t, \eta)| d \eta d \xi \\
=\left(\sum_{j=2}^{k}+\sum_{j=k+1}^{r+1}+\sum_{j=r+2}^{r+k}\right) \ldots=: I^{1}+I^{2}+I^{3} .
\end{gathered}
$$

Similarly to (3.11) and (3.12) we can obtain

$$
I^{1} \leq C_{4} \sum_{j=2}^{k}\left(\begin{array}{c}
r+k \\
j
\end{array}\right) Z_{k}(t) Z_{j-1}(t) Z_{k+1-j}(t)
$$

and

$$
I^{3} \leq C_{4} \sum_{j=r+2}^{r+k}\left(\begin{array}{c}
r+k \\
j
\end{array}\right) Z_{k}(t) Z_{j-r-1}(t) Z_{k+r+1-j}(t) .
$$

For the term $I^{2}$ in $(3.13)$, by using

$$
\mathbb{R}^{n}=\left\{|\eta| \leq C_{5}|\xi-\eta|\right\} \cup\left\{|\eta| \geq C_{5}|\xi-\eta|\right\}
$$


for some positive constant $C_{5}$, we have

$$
\begin{aligned}
& I^{2}=\sum_{j=k+1}^{r+1}\left(\begin{array}{c}
r+k \\
j
\end{array}\right)\left(\iint_{|\eta| \leq C_{5}|\xi-\eta|}+\iint_{|\eta| \geq C_{5}|\xi-\eta|}\right)\langle\xi-\eta\rangle^{r+2} \\
& \quad \times|\hat{u}(t, \xi-\eta)| \frac{\langle\xi\rangle^{r+k}|\hat{\omega}(t, \xi)|}{\langle\eta\rangle^{j-2}\langle\xi-\eta\rangle^{r+2-j}}\langle\eta\rangle^{r+k-1}|\hat{\omega}(t, \eta)| d \eta d \xi \\
& \leq C_{6} \sum_{j=k+1}^{r+1}\left(\begin{array}{c}
r+k \\
j
\end{array}\right) \iint\langle\xi\rangle^{r+k}|\hat{\omega}(t, \xi)|\left(\langle\eta\rangle^{-r}+\langle\xi-\eta\rangle^{-r}\right) \\
& \quad \times\langle\xi-\eta\rangle^{r+2}|\hat{u}(t, \xi-\eta)|\langle\eta\rangle^{r+k-1}|\hat{\omega}(t, \eta)| d \eta d \xi \\
& \leq C_{7} \sum_{j=k+1}^{r+1}\left(\begin{array}{c}
r+k \\
j
\end{array}\right) Z_{k}(t) Z_{1}(t) Z_{k-1}(t), \quad t \geq 0 .
\end{aligned}
$$

Substituting (3.14)-(3.16) into (3.13), and using (3.9) we immediately obtain (3.5).

4. Gevrey regularity for semilinear Schrödinger equations. Without loss of generality we may assume $n=1$ and $g(u)=u^{q}$ (in the general case $n>1$ and $g(u$ ) polynomial only additional technical and notational difficulties occur). We write $x$ instead of $x_{1}$ and $D$ for $\partial_{x}$. Set

$$
W_{k}=D^{k} u, \quad U_{k}(t)=\left\|W_{k}(t, \cdot)\right\|_{H^{r}}, \quad k \in \mathbb{N} .
$$

We differentiate equation (1.6) $k$ times with respect to $x$, multiply it by $W_{k}$, integrate in $H^{r}\left(\mathbb{R}^{n}\right)$. Using the Leibniz rule for differentiation, we get

$$
\begin{aligned}
\frac{1}{2} \frac{d}{d t}\left(U_{k}^{2}(t)\right) \leq & \operatorname{Re}\left(\left\langle W_{k}(t, \cdot), q u^{q-1}(t, \cdot) W_{k}(t, \cdot)\right\rangle_{H^{r}}\right) \\
& +\operatorname{Re}\left(\left\langle W_{k}(t, \cdot), R_{k}(t, \cdot)\right\rangle_{H^{r}}\right),
\end{aligned}
$$

where

$$
R_{k}(t, \cdot)=\sum_{\ell=2}^{\min \{q, k\}} \frac{q ! u^{q-\ell}(t, \cdot)}{(q-\ell) ! \ell !} \sum_{\substack{k_{1}+\ldots+k_{\ell}=k \\ k_{1} \geq 1, \ldots, k_{\ell} \geq 1}} \frac{k !}{k_{1} ! \cdots k_{\ell} !} \prod_{\mu=1}^{\ell} W_{k_{\mu}}(t, \cdot)
$$

for all $k \in \mathbb{N}$.

We recall that since $r$ is an integer greater than $n / 2$ and we consider classical Sobolev spaces $H^{r}(\Omega)$, we have the following Schauder type estimates (e.g., cf. [GR]):

$$
\left\|u^{q-1}(t, \cdot) W_{k}(t, \cdot)\right\|_{H^{r}} \leq\left(\|u(t, \cdot)\|_{L^{\infty}}\right)^{q-1} U_{k}(t)+M_{1}\left(\|u(t, \cdot)\|_{H^{r}}\right)^{q-1} U_{k-1}(t),
$$

for all $k \in \mathbb{N}, t \geq 0$, where $M_{1}=\theta^{q-1}, \theta>0$ being a positive constant in the Schauder lemma for the product in $H^{r}(\Omega)$, depending only on $n$ and $r$. Combining (4.3) with the decay estimate (1.13) for $\kappa=\infty$ and the estimate (1.14) we get that for some constant $A_{0}>0$, defined in an obvious way by the decay of $\|u(t, \cdot)\|_{L^{\infty}}$ in (1.13), the following inequality holds

$$
\left\|u^{q-1}(t, \cdot) W_{k}(t, \cdot)\right\|_{H^{r}} \leq A_{0}(1+t)^{-(q-1) \gamma} U_{k}(t)+M_{1} \delta^{q-1} U_{k-1}(t),
$$


for all $k \in \mathbb{N}, t \geq 0$. Set

$$
V_{k}(t)=\frac{U_{k}(t)}{(k !)^{\sigma}}, \quad k \in \mathbb{N} .
$$

On the other hand, using the Moser type inequality

$$
\left\|f_{1} \ldots f_{q}\right\|_{H^{r}} \leq C \sum_{\mu=1}^{q}\left\|f_{\mu}\right\|_{H^{r}} \prod_{\nu \neq \mu}\left\|f_{\nu}\right\|_{H^{r-1}}
$$

for some $C=C(q, r)>0$ and the nonlinear Gevrey estimates from [GR] we deduce that one can find a constant $M_{2}>0$ depending on $q, r$ and $M_{0}$, such that

$$
\frac{\left\|R_{k}(t, \cdot)\right\|_{H^{r}}}{(k !)^{\sigma}} \leq M_{2} \sum_{\ell=2}^{\min \{q, k\}} \delta^{q-\ell} \sum_{\substack{k_{1}+\ldots+k_{\ell}=k \\ k_{1} \geq 1, \ldots, k_{\ell} \geq 1}} \sum_{\mu=1}^{\ell} \mathcal{P}_{k_{1}, \ldots, k_{\ell}}^{\ell, \mu}(\sigma) V_{k_{\mu}}(t) \prod_{\nu \neq \mu} V_{k_{\nu}-1}(t),
$$

for every $k \in \mathbb{N}, t \geq 0$, where

$$
\mathcal{P}_{k_{1}, \ldots, k_{\ell}}^{\ell, \mu}(\sigma)=\left(\frac{k_{1} ! \cdots k_{q} !}{k !}\right)^{\sigma-1} \prod_{\nu \neq \mu} \frac{1}{k_{\nu}^{\sigma}}, \quad 2 \leq \ell \leq q
$$

with the convention $\mathcal{P}_{k_{1}}^{1,1}(\sigma)=1$. Hence, combining (4.1), (4.4) and (4.6), we obtain

$$
\begin{aligned}
V_{k}^{\prime}(t) & \leq q A_{0}(1+t)^{-\gamma(q-1)} V_{k}(t)+\frac{M_{1} \delta^{q-1}}{k^{\sigma}} V_{k-1}(t) \\
& +M_{2} \sum_{q=2}^{\min \{q, k\}} \delta^{q-j} \sum_{\substack{k_{1}+\ldots+k_{\ell}=k \\
k_{1} \geq 1, \ldots, k_{\ell} \geq 1}} \sum_{\mu=1}^{\ell} \mathcal{P}_{k_{1}, \ldots, k_{\ell}}^{\ell, \mu}(\sigma) V_{k_{\mu}}(t) \prod_{\nu \neq \mu} V_{k_{\nu}-1}(t),
\end{aligned}
$$

for every $k \in \mathbb{N}, t \geq 0$. Set

$$
Q_{k}(t):=\exp \left(-k M_{3}(1+t)^{1-\gamma(q-1)}\right) V_{k}(t)
$$

with the constant $M_{3}>0$ satisfying

$$
M_{3}(1+t)^{1-\gamma(q-1)} \geq q A_{0} \int_{0}^{t}(1+\tau)^{-\gamma(q-1)} d \tau, \quad t \geq 0
$$

Therefore, multiplying (4.8) by $\exp \left(-k M_{3}(1+t)^{1-\gamma(q-1)}\right)$ and taking into account the identity

$$
\begin{aligned}
\exp \left(-k M_{3}(1+t)^{1-\gamma(q-1)}\right) V_{k_{\mu}}(t) \prod_{\nu \neq \mu, 1 \leq \nu \leq \ell} V_{k_{\nu}-1}(t) & \\
& =\exp \left(-M_{3}(\ell-1)(1+t)^{1-\gamma(q-1)}\right) Q_{k_{\mu}}(t) \prod_{\nu \neq \mu, 1 \leq \nu \leq \ell} Q_{k_{\nu}-1}(t),
\end{aligned}
$$


we obtain

$$
\begin{aligned}
& Q_{k}^{\prime}(t) \leq \frac{M_{1} \delta^{q-1}}{k^{\sigma}} \exp (\left.-M_{3}(1+t)^{1-\gamma(q-1)}\right) Q_{k-1}(t) \\
&+M_{2} \sum_{\ell=2}^{\min \{q, k\}} \exp \left(-M_{3}(\ell-1)(1+t)^{1-\gamma(q-1)}\right) \delta^{q-\ell} \\
& \times \sum_{\substack{k_{1}+\ldots+k_{\ell}=k \\
k_{1} \geq 1, \ldots, k_{\ell} \geq 1}} \sum_{\mu=1}^{\ell} \mathcal{P}_{k_{1}, \ldots, k_{\ell}}^{\ell, \mu}(\sigma) Q_{k_{\mu}}(t) \prod_{\nu \neq \mu} Q_{k_{\nu}-1}(t) \\
& \leq \frac{M_{1} \delta^{q-1}}{k^{\sigma}} \exp \left(-M_{3}(1+t)^{1-\gamma(q-1)}\right) Q_{k-1}(t) M_{2} \sum_{\substack{\ell=2 \\
\min \{q, k\}}}^{\exp \left(-M_{3}(\ell-1)(1+t)^{1-\gamma(q-1)}\right) \delta^{q-\ell}} \\
& \times \sum_{k_{1}+\ldots+k_{\ell}=k}^{\ell} \sum_{\mu=1}^{\ell} \mathcal{P}_{k_{1}, \ldots, k_{\ell}}^{\ell, \mu}(\sigma) Q_{k_{\mu}}(t) \prod_{\nu \neq \mu} Q_{k_{\nu}-1}(t) .
\end{aligned}
$$

We observe that by the definition of the constant $A_{0}$ we get that $A_{0}=O\left(\left(\left\|u_{0}\right\|_{H^{r}}\right)^{q-1}\right)$. Since the right-hand side of (4.10) does not depend on $Q_{k}(t)$, we derive (1.18) by arguments similar to (and easier than) the ones used in Section 3. We show in details (1.19) since somewhat more subtle estimates are involved. Choose and fix $0<\varepsilon \ll 1$. Then for every small positive number $0<\varepsilon_{1} \ll \varepsilon$ we have the freedom to choose $\delta \ll \varepsilon_{1}$. We set $\mathcal{Q}_{k}^{\varepsilon}(t)=Q_{k}(t) \exp (-k \varepsilon t)$ and

$$
\mathcal{B}_{N}^{\varepsilon}:=\max _{1 \leq k \leq N} \sup _{t \geq 0} \mathcal{Q}_{k}^{\varepsilon}(t), \quad N \in \mathbb{N} .
$$

Then, taking into account (4.7) and (4.10), we can find a positive number $M_{4}$ depending on $M_{1}, M_{2}, n, q$ and $\sigma$, such that

$$
\begin{aligned}
\mathcal{B}_{N}^{\varepsilon} & \leq\left\|u_{0}\right\|_{\sigma, \rho, H^{r}}+\frac{M_{4}}{N^{1+\sigma} \varepsilon} \sum_{j=1}^{q} \delta^{q-j}\left(\mathcal{B}_{N-1}^{\varepsilon}\right)^{j} \\
& \leq\left\|u_{0}\right\|_{\sigma, \rho, H^{r}}+\frac{M_{4}}{N^{1+\sigma}} \frac{\delta}{\varepsilon} \sum_{j=1}^{q-1} \delta^{q-j-1}\left(\mathcal{B}_{N-1}^{\varepsilon}\right)^{j}+\frac{M_{4}}{N^{1+\sigma} \varepsilon}\left(\mathcal{B}_{N-1}^{\varepsilon}\right)^{q}, \quad N \in \mathbb{N} .
\end{aligned}
$$

Note that problems in dealing with (4.12) might occur for $N^{1+\sigma} \leq O\left(\varepsilon^{-1}\right)$. At this point we choose $\delta \ll \varepsilon_{1}, \varepsilon_{1} \ll \min \left\{\left\|u_{0}\right\|_{\sigma, \rho, H^{r}}, \varepsilon\right\}$ so that both $\delta \varepsilon^{-1}$ and the $H^{N}(\Omega)$ norms of $u(t, \cdot)$ for $N^{1+\sigma} \leq O\left(\varepsilon^{-1}\right)$ become small enough. If $N^{1+\sigma} \gg O\left(\varepsilon^{-1}\right)$, then the convergence of $\left\{\mathcal{B}_{N}^{\varepsilon}\right\}$ for $N \rightarrow+\infty$ follows by iteration type arguments as for (2.14). The proof of (1.19) is complete.

5. Concluding remarks. We begin by pointing out that if $\Omega=\mathbb{T}^{2}$ and $\sigma=1$, the decay of the Gevrey $G^{1}$ radius $\rho_{\sigma}(t)$ in (1.16) is slower than the decay shown in Theorem 7 of $[\mathrm{LO}]$. Indeed, by $(\mathrm{H} 3)$ we obtain that

$$
\|\nabla K[\omega(t, \cdot)]\|_{H^{r}} \leq C\|\omega(t, \cdot)\|_{H^{r}}=: \theta(t), \quad t \geq 0 .
$$


Hence, by (1.16), we get

$$
\rho_{1}(t) \geq \rho \exp \left(-c_{0} t\left\|\omega^{0}\right\|_{\sigma, \rho, H^{r}}-c_{0} C \int_{0}^{t} \theta\left(t_{1}\right) d t_{1}\right), \quad t \geq 0 .
$$

The decay rate for $\rho_{1}(t)$ given by the formulas $(22)$ and (24) on p. 329 of [LO] could be rewritten in the following form: there exist two positive constants $c_{1}$ and $c_{2}$ such that

$$
\rho_{1}(t)=\rho \exp \left(-c_{2} \int_{0}^{t}\left(\sqrt{\left\|\omega^{0}\right\|_{\sigma, \rho, H^{r}}^{2}+c_{1} \int_{0}^{t_{2}} \theta^{3}\left(t_{1}\right) d t_{1}}\right) d t_{2}\right), \quad t \geq 0
$$

which is faster than the decay rate given by (5.1).

We mention also that we can derive estimates for the decay of $\rho_{\sigma}(t)$ for $t \rightarrow+\infty$ in (1.15) for solutions $\vec{v}=\left(v_{1}, \ldots, v_{m}\right)$ to IVP for $m \times m$ systems of evolution equations of the type

$$
\partial_{t} \vec{v}+\sum_{j}^{n} K_{j}[\vec{v}] \partial_{x_{j}} \vec{v}+F[\vec{v}]=\vec{f}(t, x),
$$

where $F$ is an analytic nonlinear term, $\vec{f} \in C\left(\left[0,+\infty\left[:\left(G_{u n}^{\sigma}(\Omega)\right)^{m}\right), K_{j}, j=1, \ldots, n\right.\right.$, are matrix-valued operators (they might be nonlocal, as in Kirchhoff type equations $\left.u_{t t}-M\left(\|\nabla u(t, \cdot)\|_{L^{2}}^{2}\right) \Delta u=0, M \in C^{1}(\mathbb{R}:] 0,+\infty[)\right)$. The condition (H2) is replaced by the requirement

$$
\operatorname{Re}\left(\sum_{j}^{n}\left\langle v, K_{j}[v] \partial_{x_{j}} v\right\rangle\right) \geq 0, \quad v \in H^{1}(\Omega)
$$

Note that (1.2) can be reduced to an equation of the type (5.3), setting $v(t, x)=$ $b(x) \omega(t, x)$ and multiplying (1.2) by $b(x)$. The proofs become more involved from the point of view of the nonlinear analysis type difficulties.

As to the possibility to investigate the propagation of the uniform $G^{\sigma}$ regularity of solutions to initial boundary value problems (Dirichlet or Neumann type) when $\Omega \subset \mathbb{R}^{n}$ is a domain whose boundary $\partial \Omega$ is a real analytic submanifold, we observe that even for polynomial nonlinearities $g(u)$ no estimates in Banach spaces of Gevrey functions are available, as far as we know. In particular, it is necessary to analyze carefully Gevrey norms of products of eigenfunctions of $-\Delta$ in domains with zero Dirichlet or Neumann boundary conditions.

As to generalizations of Theorem 2, we can show, by the same body of ideas (iterative inequalities and precise Gevrey nonlinear estimates) but with technically more involved proofs, similar results on the uniform Gevrey regularity of solutions to Cauchy problems for the nonlinear wave equation, the Klein-Gordon equation as well as for some Schrödinger type operators with multiple characteristics (cf. [GNT], [Ta]), with quasilinear nonlinear terms depending analytically on $u$ and $\nabla u$. As for the case of nonanalytic Gevrey $G^{\sigma}, \sigma>1$, nonlinearities, it seems that some of the recent methods for obtaining nonlinear Gevrey estimates of Gevrey (but not analytic) nonlinear compositions (cf. [GR], [BRS]) might be applied for semilinear equations with Gevrey nonlinearities.

Acknowledgements. The authors thank K. Kajitani and M. Reissig for useful discussions on problems related to the Gevrey regularity for evolution equations. Part 
of the research was carried out when the first author was visiting the University of Tsukuba (February-March 2001) and when the second author was visiting the University of Cagliari (November 1999-February 2000).

\section{References}

[AM] S. Alinhac, G. Metivier, Propagation de l'analyticité locale pour les solutions de l'équation d'Euler, Arch. Rational Mech. Anal. 92 (1986), 287-296.

[Ba] C. BARdos, Existence et unicité de la solution de l'équation d'Euler en dimension deux, J. Math. Anal. Appl. 40 (1972), 769-790.

[BB] C. Bardos, S. Benachour, Domaine d'analycité des solutions de l'équation d'Euler dans un ouvert de $\mathbb{R}^{n}$, Ann. Scuola Norm Sup. Pisa Cl. Sci. (4) 4 (1977), 647-687.

[BG] H. A. Biagioni, T. GRAmchev, Evolution PDE with elliptic dissipative terms: critical index for singular initial data, self-similar solutions and analytic regularity, C. R. Acad. Sci. Paris Sér. I Math. 327 (1998), 41-46.

[BRS] G. Bourdaud, M. Reissig, W. Sickel, Hyperbolic equations, function spaces with exponential weights and Nemytskij operators, Prépublication 302, Institut de Mathématiques de Jussieu, Université de Paris VI et Paris VII, 2001;

http://www.institut.math.jussieu.fr/ preprints/index-2001.html

[CZ] M. Cicognani, L. Zanghirati, Analytic regularity for solutions of nonlinear weakly hyperbolic equations, Boll. Un. Mat. Ital. B (7) 11 (1997), 643-679.

[DS] P. D'Ancona, S. Spagnolo, Global solvability for the degenerate Kirchhoff equation with real analytic data, Invent. Math. 108 (1992), 247-262.

[FeT] A. Ferrari, E. Titi, Gevrey regularity for nonlinear analytic parabolic equations, Comm. Partial Differential Equations 23 (1998), 1-16.

[FT] C. Foias, R. Temam, Gevrey class regularity for the solutions of the Navier-Stokes equations, J. Funct. Anal. 87 (1989), 359-369.

[GG] D. Gourdin, M. Ghedamsi, Global smooth solutions for quasi-linear hyperbolic systems of second order, Commun. Appl. Anal. 5 (2001), 335-349.

[GNT] D. Gourdin, S. Ngnosse, J. Takeuchi, Problème de Cauchy pour certaines équations du type de Schrödinger, C. R. Acad. Sci. Paris Sér. I Math. 324 (1997), 1111-1116.

[GR] T. Gramchev, L. Rodino, Gevrey solvability for semilinear partial differential equations with multiple characteristics, Boll. Unione Mat. Ital. Sez. B Artic. Ric. Mat. (8) 2 (1999), 65-120.

[Kj1] K. KaJITANI, Global real analytic solutions of the Cauchy problem for linear differential equations, Comm. Partial Differential Equations 11 (1986), 1489-1513.

[Kj2] K. Kajitani, Propagation of analyticity of solutions to the Cauchy problem for Kirchhoff type equations, in: Journées "Équations aux Dérivées Partielles" (La Chapelle sur Erdre, 2000), Exp. No. VIII, 14 pp., Univ. Nantes, Nantes, 2000.

[KY1] K. Kajitani, K. Yamaguti, On global real analytic solutions of the degenerate Kirchhoff equation, Ann. Scuola Norm. Sup. Pisa Cl. Sci. (4) 21 (1994), 279-297.

[KY2] K. Kajitani, K. Yamaguti, Propagation of analyticity of the solutions to the Cauchy problem for nonlinear symmetrizable systems, Ann. Scuola Norm. Sup. Pisa Cl. Sci. (4) 28 (1999), 471-487.

[Ka] T. KATo, On classical solutions to two-dimensional nonstationary Euler equation, Arch. Rational Mech. Anal. 25 (1967), 188-200. 
[KP] S. Klainerman, G. Ponce, Global, small amplitude solutions to nonlinear evolution equations, Comm. Pure Appl. Math. 36 (1983), 133-141.

[LO] C. D. Levermore, M. Oliver, Analyticity of solutions for a generalized Euler equation, J. Differential Equations 133 (1997), 321-339.

[LOT] C. D. Levermore, M. Oliver, E. S. Titi, Global well-posedness for models of shallow water in a basin with a varying bottom, Indiana Univ. Math. J. 45 (1996), 479-510.

[Ol] M. Oliver, Classical solutions for a generalized Euler equation in two dimensions, J. Math. Anal. Appl. 215 (1997), 471-484.

[Ra] R. RACKE, Lectures on Nonlinear Evolution Equations, Aspects Math. E19, Vieweg, Braunschweig, 1992.

[RY] M. Reissig, K. YAGDJian, Levi conditions and global Gevrey regularity for the solutions of quasilinear weakly hyperbolic equations, Math. Nachr. 178 (1996), 285-307.

[Ro] L. Rodino, Linear Partial Differential Operators in Gevrey Spaces, World Scientific, Singapore, 1993.

[Sp] S. Spagnolo, Some results of analytic regularity for the semi-linear weakly hyperbolic equations of the second order, Rend. Sem. Mat. Univ. Politec. Torino, Fascicolo speciale (1988), 203-229.

[Ta] J. TAKeuchi, A necessary condition for $H^{\infty}$-wellposedness of the Cauchy problem for linear partial differential operators of Schrödinger type, J. Math. Kyoto Univ. 25 (1985), $459-472$. 